\title{
Sédimentologie, paléontologie et paléoenvironnements côtiers de la région de Porrentruy (Sud-Rhénan, Paléogène, Jura, Suisse): Implications géodynamiques
}

\author{
Laurent Picot $^{1}$, Damien Becker ${ }^{2,3}$, Fréderic Lapaire ${ }^{3}$, KAmil Ustaszewski $^{4}$, Wolfgang A. Hug ${ }^{2,3}$ \\ \& JEAN-PIERRE BERGER ${ }^{2}$
}

Key words: Molasse, Rhinegraben, sedimentology, paleontology, Rupelian, paleoecology, tectonic

\section{ABSTRACT}

Located in the distal part of the Swiss Molasse Basin and in the southern extension of the Rhine Graben, the conglomeratic deposits belonging to the Gompholithes \& Conglomérats stratigraphic group have been the object of detailed sedimentological and paleontological studies. The great number of outcrops that came into sight during the building works of the Transjurane highway in the vicinity of Porrentruy (Swiss Jura) lead to a better understanding of Rupelian paleoenvironments (Early Oligocene).

The sedimentological and paleontological studies reveal the existence of coastal environments with Mesozoic limestone cliffs notched by canyons with torrential rivers. Those rivers eroding the Mesozoic series create pebbles deposits forming marine deltas prograding towards North. In protected areas, some lacustrine environments can develop.

These conglomeratic deposits are strongly bound to the Rupelian tectonic activity. The rhenish distension and the activity of the transform faults located between the Rhine Graben and the Bresse basin divide the Mesozoic blocks in horst and graben structures, thus allowing the erosion of sediments in higher regions (horst) and their transport in lower zones (graben).

The discovery of rare pebbles made of endogene and effusive rocks in those conglomeratic deposits shows a transport coming from the Vosges massifs towards south to the Porrentruy region, probably with the support of a littoral drift. Although the surrection of the Vosges and Schwarzwald massifs (and the beginning of their erosion) is normally attributed to the base of the Miocene, the presence of those pebbles attests the existence of faults putting the basement of the Vosges massif to erosion since the base of Rupelian.

\section{RESUME}

Situés dans la partie la plus distale du bassin molassique suisse et dans le prolongement sud du fossé rhénan, les dépôts conglomératiques appartenant au groupe stratigraphique des Gompholithes \& Conglomérats ont fait l'objet d'une étude sédimentologique et paléontologique détaillée. La multitude des affleurements réalisés lors des travaux de construction de l'autoroute Transjurane dans la région de Porrentruy (Jura), permet d'appréhender ces paléoenvironnements rupéliens (Oligocène inférieur).

Les études sédimentologiques et paléontologiques révèlent l'existence d'environnements côtiers avec des falaises de calcaires mésozoïques entaillées par des canyons où se trouvent des rivières au régime torrentiel. Ces rivières qui érodent les couches du Mésozoïque créent des galets qui sont déposés sous la forme de deltas marins progradant vers le nord. A l'abri des exutoires des canyons se développent quelques environnements lacustres.
Ces dépôts conglomératiques sont fortement liés à l'activité tectonique rupélienne. La distension rhénane et l'activité de la faille transformante située entre le fossé rhénan et le bassin de la Bresse subdivisent les différents blocs mésozoïques en horsts et grabens, permettant ainsi l'érosion des sédiments dans les parties hautes (horst) et leur transport dans les zones basses (graben).

La découverte de rares galets de roches endogènes et effusives dans les dépôts conglomératiques montre un transport du socle des Vosges vers le sud dans la région de Porrentruy par l'intermédiaire sans doute d'une dérive littorale. Bien que la surrection des Vosges et de la Forêt-Noire et leur mise à l'érosion soient connues dès la base du Miocène, la présence de ces galets atteste l'existence de failles dès le début du Rupélien qui mettent à l'érosion le socle du massif des Vosges.

\section{ZUSAMMENFASSUNG}

Im distalsten Teil des schweizerischen Molasse-Beckens und in der Südverlängerung des Rheingrabens befinden sich die Konglomeratablagerungen der stratigraphischen Gruppe Gompholithes \& Conglomérats, die hier einer sedimentologischen und paläontologischen detaillierten Studie unterzogen werden. Die zahlreichen Aufschlüsse, die beim Bau der Autobahn Transjurane in der Region von Porrentruy (Jura) zu Tage getreten sind, erlauben, diese rupelische (fruhes Oligozän) Paläoumgebung besser zu verstehen.

Die sedimentologischen und paläontologischen Studien deuten auf die Existenz einer Küste aus mesozoischen Kalkklippen hin, die von Cañons eingeschnitten wurde. Sintflutartige Flüsse, die das Mesozoikum erodierten, Gerölle sedimentierten, die in einem nach Norden progradierenden Delta in das Meer geschüttet wurden. Lokal entwickelte sich eine lakustrine Umgebung mit kontinentalen Kalksedimenten.

Diese Konglomeratablagerungen entstanden im Zusammenhang mit der tektonischen Aktivität im Rupelium. Die Rheingrabendehnung und die Aktivität der Blattverschiebungen zwischen dem Rheingraben und dem BresseBecken führten zur Zergliederung der mesozoischen Blöcke in Horste und Gräben. Es resultierte die Erosion der Sedimente in den gehobenen Teilen (Horste) und ihr Transport respektive Ablagerung in die niedrigen Zonen (Gräben).

Die Nachweis von Geröllen endogener und effusiver Gesteine in den Konglomeratablagerungen weist auf einen Transport aus dem Vogesen durch Küstenströmungen in Richtung Süden hin in die Region von Porrentruy und auf die Herkunft der Gerölle aus dem Vogesen Massiv. Die Anhebung der Vogesen und des Schwarzwaldes und der Beginn ihrer Erosion wurde bislang mit Unter-Miozäns datierte. Unsere Untersuchungen belegen die Existenz von Brüchen, und den Beginn der Erosion schon an der Basis des Rupelium.

\footnotetext{
${ }^{1}$ Département Géosciences, UFR Sciences et Techniques, 16, route de Gray 25030 Besançon cedex, France.

${ }^{2}$ Institut de Géologie et de Paléontologie, Université de Fribourg, Pérolles, 1700 Fribourg, Suisse. Email: jean-pierre.berger@unifr.ch

${ }^{3}$ Section de Paléontologie, Hôtel des Halles, Case Postale 64, 2900 Porrentruy, Suisse.

${ }^{4}$ Geologisch-Paläontologisches Institut, Universität Basel, Bernoullistrasse 32, 4056 Basel, Suisse.
} 


\section{Introduction}

\subsection{Localisation}

Le bassin molassique suisse est un bassin d'avant-pays classique (Homewood et al. 1986, Berger 1989). Dans sa partie proximale, la Molasse subalpine représente des dépôts plissés et faillés, alors que plus au nord, la Molasse du Plateau est constituée de dépôts très épais (plusieurs kilomètres) à faible pendage. La zone d'étude se situe dans la partie la plus distale du bassin molassique suisse, constituée de dépôts généralement plissés de faibles épaisseurs piégées dans les synclinaux du Jura. Cette zone se trouve dans le prolongement sud du fossé rhénan bordé à l'ouest et à l'est par le massif des Vosges et celui de la Forêt-Noire. Elle fait donc le lien entre le Tertiaire de la bordure sud-rhénane et le Tertiaire des synclinaux du Jura (Fig. 1). Le Paléogène jurassien a fait l'objet d'une thèse récente (Picot 2002) qui reprend notamment toute la littérature existante de manière exhaustive. Pour cette raison, les citations bibliographiques seront réduites ici à leur strict minimum.

Dans la région de Porrentruy (Atlas géologique au 1:25 000, 1085 St-Ursanne, Diebold et al. 1963), différentes coupes et forages ont été étudiés permettant une bonne compréhension des paléoenvironnements des dépôts tertiaires. Les principaux affleurements étudiés sont (Fig. 1):

la coupe de Bressaucourt Bois Carré (BRE), (coord. 571.3 / 250.35)

la coupe de Bressaucourt UMM (BREU), (coord.571.55 / 251.2)

le forage de l'Etang (ETA), (coord. 571.474 / 251.036)

la coupe de l'Oiselier (passage à grenouilles) (OIP), (coord. 571.550 / 250.4)

la coupe de l'Oiselier (sortie du tunnel) (OIS), (coord. $571.85 / 250.5)$

le forage de la Vauche (RAV), (coord. 572.832 / 251.53)

\subsection{Stratigraphie}

Les coupes de Bressaucourt Bois Carré (BRE), de l'Oiselier (passage à grenouilles; OIP), de l'Oiselier (sortie du tunnel; OIS) ainsi que la base des forages de l'Etang (ETA) et de la Vauche (RAV) sont caractérisées par des dépôts conglomératiques surmontant les dépôts calcaires mésozoïques majoritairement kimméridgiens. Les galets des dépôts conglomératiques cénozoïques sont essentiellement constitués de calcaires oxfordiens et kimméridgiens provenant de la région. Quelques rares galets de roches endogènes et effusives ont été observés dans un niveau conglomératique de la coupe de l'Oiselier (passage à grenouilles). Les dépôts conglomératiques peuvent parfois être interrompus par des niveaux calcaires ou marneux.

Schneider (1960) nomme Conglomérats de Porrentruy la formation qui comprend les restes de conglomérats d'eau douce contenant du Bolus et des pisolithes de fer remaniés, plus vieux que le Rupélien marin, et qui se trouvent dans un couloir NE-SW creusé dans le Malm en passant par Bressaucourt et Porrentruy. Il considère que ces dépôts sont exclusivement fluviatiles. L'analyse paléontologique de ce travail montre que certains niveaux sont marins, ce qui nuance le propos de Schneider. Dans la région de Porrentruy, la formation de la Gompholithe d'Ajoie, définie par Liniger (1925) dans le synclinal voisin de Delémont, est une formation constituée de dépôts conglomératiques contenant quelques fossiles marins (Rollier 1893, Birkhäuser 1925, Baumberger 1934). L'appartenance de ces dépôts conglomératiques à une des deux formations se basait donc essentiellement sur la présence ou l'absence de fossiles marins, qui par ailleurs sont facilement détruits mécaniquement dans ce type d'environnement.

A la lumière des nouvelles découvertes de faunes marines dans la formation des Conglomérats de Porrentruy, et d'une étude synthétique de toutes les formations paléogènes des synclinaux du Jura, Picot (2002) a redéfini ce groupe lithostratigraphique. Il regroupe sous le terme Gompholithe et Conglomérats tous les conglomérats fluviatiles et marins, semblant souvent représenter des environnements côtiers. Ce groupe comprend les formations des Conglomérats de Porrentruy (Schneider 1960), des Gompholithes d'Ajoie (Rollier 1893 Liniger 1925), ainsi que la formation des Calcarénites d'Oltingue (Fischer 1965a et b). Les Gompholithes \& Conglomérats reposent directement sur les dépôts du Mésozoïque et sur le Sidérolithique.

L'affleurement de Bressaucourt UMM (BREU) et le sommet du forage de l'Etang (ETA) montrent des marnes grises à beiges lorsqu'elles sont oxydées. Ces marnes marines riches en microfossiles appartiennent au groupe stratigraphique du Septarienton (Picot 2002).

\section{Etude sédimentologique et paléontologique des différentes coupes}

\subsection{La coupe de Bressaucourt Bois Carré (BRE), (coord. 571.3/250.35)}

A la faveur de la réfection de la route entre Porrentruy et Bressaucourt, nous avons levé une coupe composée de calcaires, marnes, grès et conglomérats d'une puissance de plus de 30 m, à l'est du lieu-dit Bois Carré (coord. 571.30/ 250.35). Cet affleurement montre un pendage de 20 à 25\% 330-335.

La coupe de Bois Carré (Fig. 2) se situe à quelques mètres au-dessus des couches mésozoïques, sans que le contact soit visible.

Elle montre, à sa base, des bancs calcaires légèrement marneux de plus de $2 \mathrm{~m}$ d'épaisseur. Ces bancs calcaires contiennent, à la base, des concrétions calcitiques blanchâtres d'un demi-centimètre et au sommet du premier banc un niveau conglomératique avec des galets d'un diamètre allant de 5 à 20 $\mathrm{cm}$. Les galets proviennent des formations du Mésozoïque local.

Ces niveaux calcaires sont surmontés par près de $8 \mathrm{~m}$ de marnes grises, bariolées et sombres. Ces marnes contiennent 


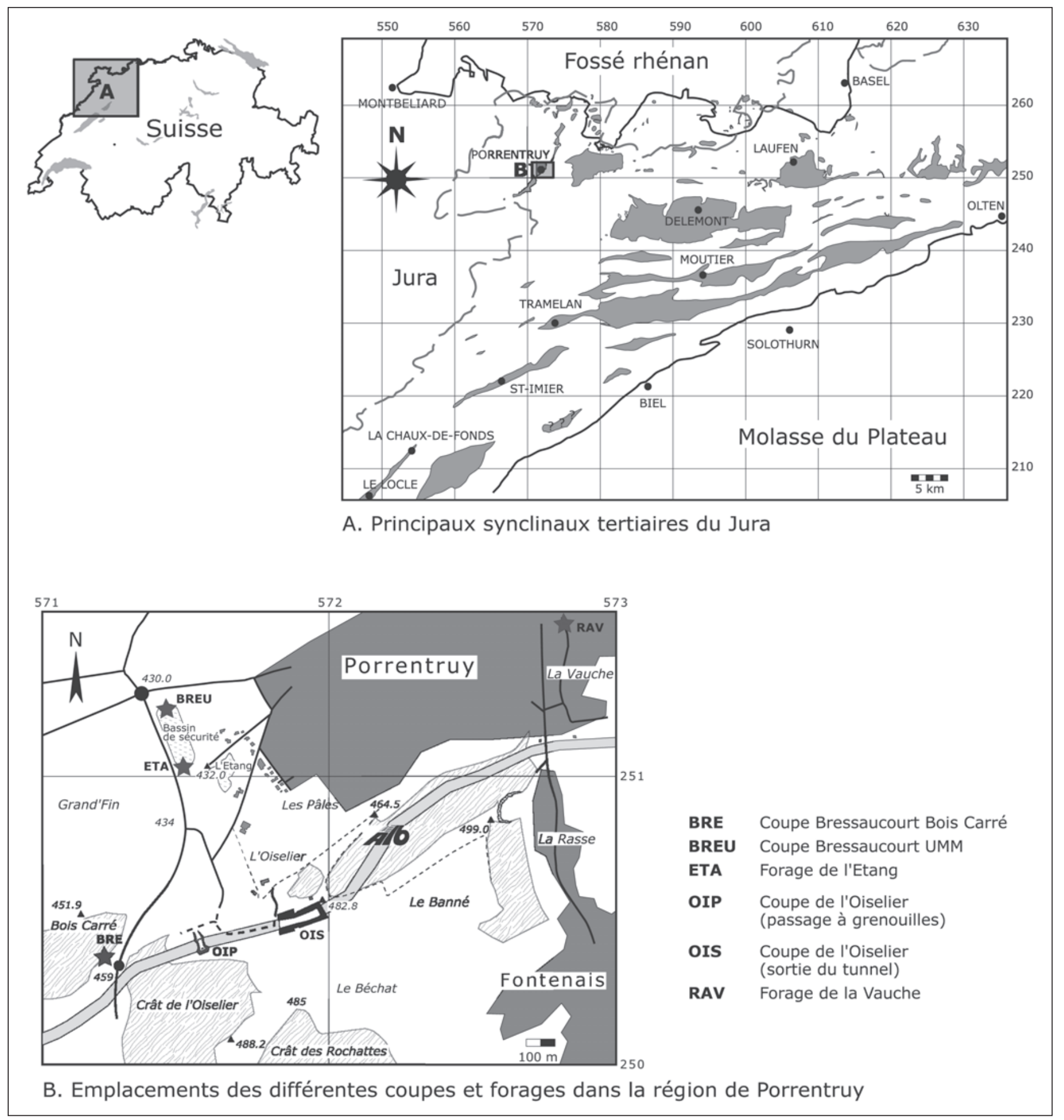

Fig. 1. Localisation de la zone d'étude et des différentes coupes étudiées.

par endroits des niveaux discontinus de galets d'origine jurassique allant jusqu'à un diamètre de $5 \mathrm{~cm}$; le banc BRE 5 montre un niveau avec des concrétions. Cette série marneuse est surmontée d'un grès fin montrant des traces racinaires, synonymes d'émersion.
Surmontant la série marneuse et jusqu'au sommet de la coupe, les dépôts se caractérisent par une succession de conglomérats entrecoupée d'épisodes gréseux et marneux. Les niveaux conglomératiques se caractérisent par une base érosive ainsi que par la présence de galets non triés allant d'un dia- 


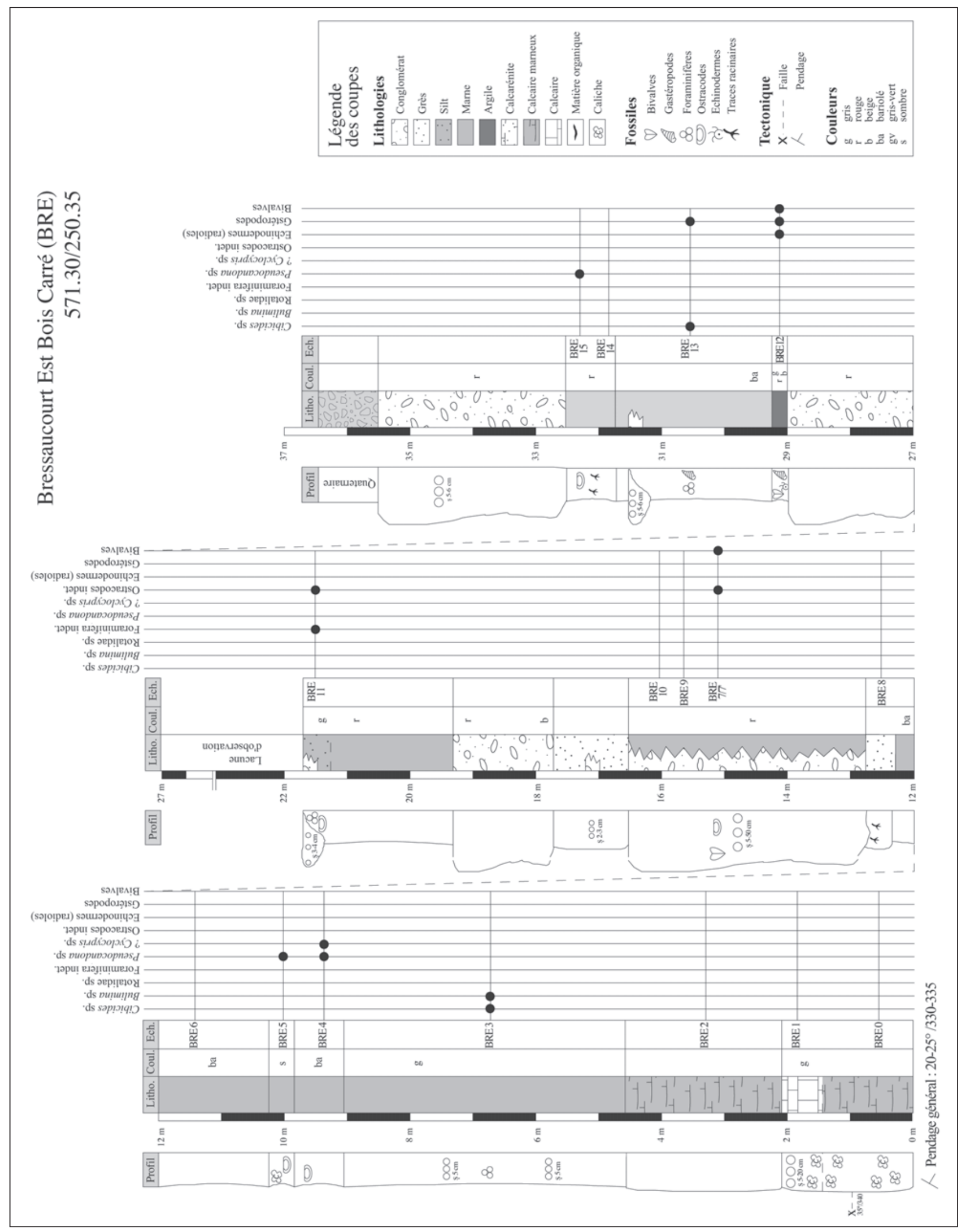


mètre de $5 \mathrm{~cm}$ à $60 \mathrm{~cm}$. Le niveau BRE 7 montre également des remaniements de brèches, ainsi que des blocs aux surfaces arrondies et polies d'environ $1 \mathrm{~m}$ de diamètre. La nature des galets des conglomérats est majoritairement composée de calcaires mésozoïques, mais de rares galets de quartzite rouge sont présents. Le niveau BRE $7 / 7^{`}$ montre une variation latérale de faciès, à savoir d'une part un conglomérat à matrice composée d'argiles sableuses rouges, et d'autre part des marnes rouges. Le conglomérat forme un chenal dans ces marnes rouges. Ces chenaux sont de grande taille (plus de $4 \mathrm{~m}$ de haut). La matrice des différents niveaux conglomératiques est constituée de marnes plus ou moins gréseuses, souvent de couleur rouge. Dans les intercalations marneuses, on peut observer des petits chenaux d'1.50 m de large pour une puissance de 50 à $60 \mathrm{~cm}$ (BRE 11 et BRE 13). Ces petits chenaux contiennent un conglomérat dont les galets atteignent un diamètre maximal de $6 \mathrm{~cm}$. Les niveaux BRE 14 et BRE 15 montrent des traces racinaires, ainsi que des manchons calcitiques, synonymes de construction microbienne sur la base des végétaux (Duringer \& Gall 1994).

La base de la coupe se distingue par une faible énergie, permettant le dépôt des calcaires et des marnes. A l'opposé, le sommet de la coupe se caractérise par une très haute énergie (des blocs d'1 m sont transportés), où se mettent en place des chenaux conglomératiques passant latéralement à des marnes ou des grès.

Des lames minces ont été effectuées dans les niveaux calcaires à la base de la coupe. Les niveaux BRE 0 et BRE 1 montrent des calcaires micritiques à structure globuleuse et micropéloïdale. La structure globuleuse est due à différentes phases de dissolution et de précipitation, ainsi qu'à la précipitation de sparite le long des racines, où se trouvent les circulations préférentielles de fluides riches en carbonate. La dissolution de fossiles tels que des gastéropodes ou des cavités dues à des racines ont permis l'installation de ces cristaux à la périphérie des cavités (Freytet \& Plaziat 1982). La lame du niveau BRE 0 montre une croissance globulaire de sparite en plusieurs couches. Cette structure est caractéristique de tuf ou de travertin. Les travertins actuels sont riches en aragonite, mais ce minéral recristallise ensuite en calcite. L'aspect caverneux est dû en partie à la disparition de débris végétaux encroûtés de carbonates d'origine biochimique (activité d'algues bleues). Une lame mince effectuée dans le niveau calcaire BRE 2 montre que ce calcaire est micritique à structure péloïdale, et contient de rares quartz. De nombreuses cavités dûes à des dissolutions sont remplies de cristaux de sparite, créant un ciment drusique.

Certains sédiments provenant des niveaux fins de la coupe ont été lavés pour y récolter les flores et les faunes. En raison de la nature des sédiments et de la haute énergie de ces dépôts, peu de fossiles ont été conservés et les différents niveaux ont révélé une faune assez pauvre.

Les niveaux BRE 3, BRE 11 et BRE 13 ont fourni une faune de foraminifères tertiaires (déterminations Kirsten Grimm). Le niveau BRE 3 a fourni Bulimina sp. et des Rotali- dae indéterminables. Le niveau BRE 13 a fourni Cibicides sp. Un autre niveau, BRE 11, a fourni des foraminifères, mais ils ne peuvent être déterminés car ils sont recristallisés.

Les ostracodes récoltés dans les sédiments de cette coupe sont tous continentaux. Le niveau BRE 4 a fourni les espèces Pseudocandona sp.1 et ?Cyclocypris sp. Le niveau BRE 5 a livré Pseudocandona sp.1. Les niveaux BRE 7 et BRE 11 ont livré des fragments de carapace indéterminables. Ces ostracodes, typiquement continentaux, vivent dans des tranches d'eau faibles (0-2 m pour le genre Pseudocandona). Les Pseudocandona peuvent également supporter des eaux légèrement salines (Apostolescu \& Guernet 1992).

Le niveau BRE 12 a fourni des radioles d'oursins. En raison de leur mauvaise conservation, nous ne pouvons dire s'ils sont autochtones, ou s'ils ont été remaniés des dépôts du Jurassique.

Les niveaux BRE 12 et BRE 13 ont livré des gastéropodes. D'une taille entre 250 et $500 \mu \mathrm{m}$, l'assemblage est monospécifique.

Les niveaux BRE 7 et BRE 12 ont livré des fragments de bivalves. En raison de leur mauvaise conservation, nous ne pouvons dire s'ils sont autochtones, ou s'ils ont été remaniés du Jurassique.

\subsection{La coupe de Bressaucourt UMM (BREU), (coord. 571.55 / 251.2)}

La coupe de Bressaucourt UMM (BREU) est un petit affleurement temporaire qui se situe à l'ouest de Porrentruy (coord. 571.55 / 251.20). Cette mini-coupe, affleurant lors de la construction de réservoirs d'eau montre à sa base $20 \mathrm{~cm}$ de marnes grises contenant de petits bivalves (niveau BREU 1), surmontés de $60 \mathrm{~cm}$ d'argiles brunes avec quelques passées plus claires (niveau BREU 2). Le remblai de cette excavation a été entreposé non loin de là, et en raison de sa riche macrofaune, il a été échantillonné pour un lavage (BREU remblai).

Peu d'indices sédimentologiques ont été relevés. Il s'agit de dépôts marneux de basse énergie, où s'étendent des fronts d'oxydation le long des petites fractures.

Les lavages effectués dans les niveaux de la coupe ont révélé une riche faune.

Les deux niveaux de la coupe et l'échantillon provenant du remblai ont tous fournis de petits bivalves, dans toutes les fractions des tamis. Ces bivalves peuvent être séparés en deux groupes: le premier est constitué de petits bivalves à charnière hétérodonte et montre, en vue interne, une empreinte sinupalliée. La taille des bivalves et leur conservation ne permettent pas d'apprécier les empreintes musculaires. En vue externe, la surface de la coquille est lisse et ne laisse apparaître que les stries d'accroissement. Puisque les coquilles montrent des empreintes sinupalliées, il s'agit sans doute d'une espèce endobionte, qui ressemble beaucoup aux Tellines actuelles. Le deuxième groupe est représenté par des valves à charnière hétérodonte. L'état de conservation ne permet pas d'apprécier l'empreinte du manteau, ni l'emplacement des muscles. En vue 


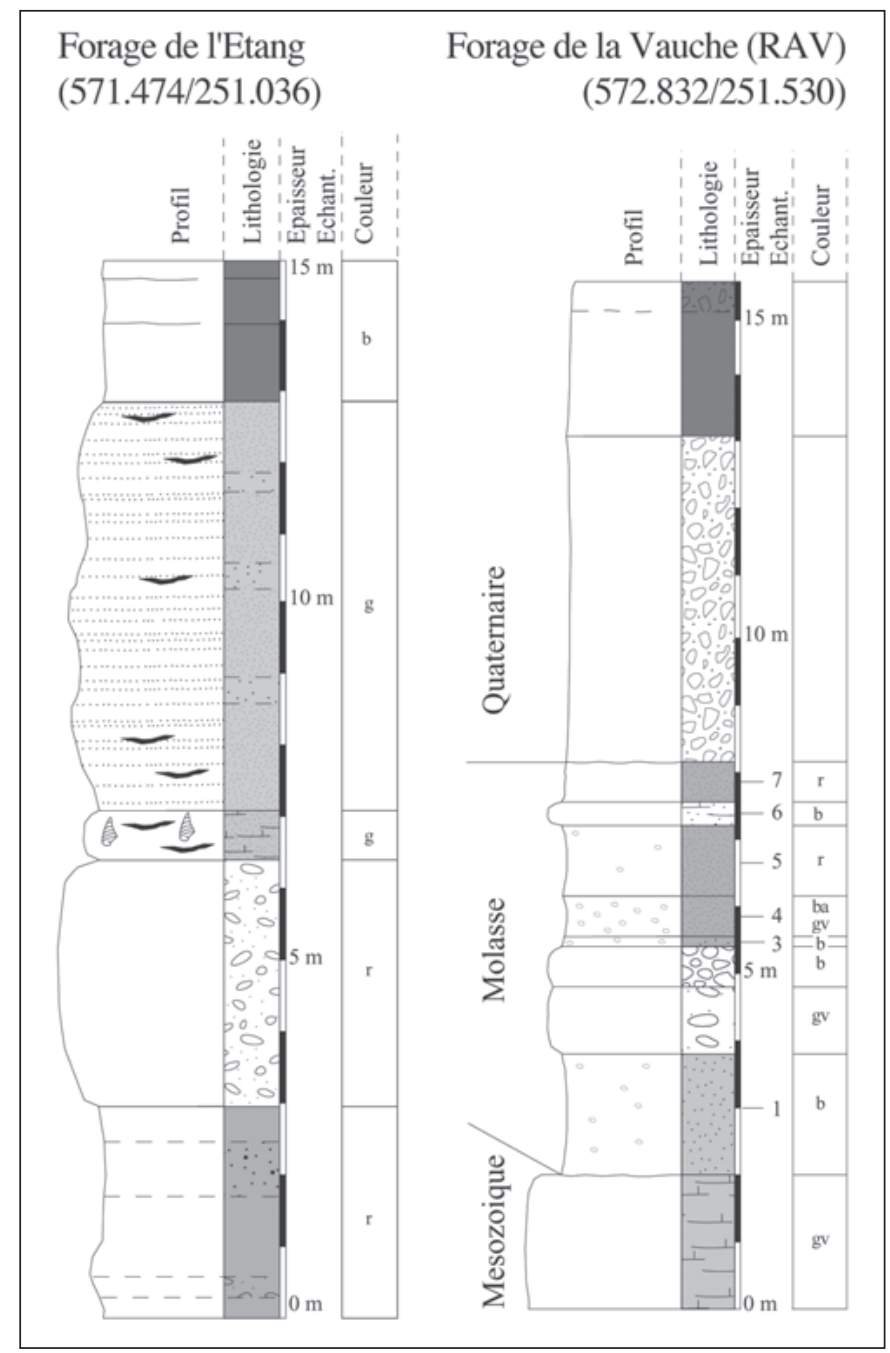

Fig. 3. Logs lithostratigraphiques des forages de l'Etang (ETA) et de la Vauche (RAV); pour la légende voir Fig. 2.

externe, la coquille porte des côtes perpendiculaires aux stries d'accroissement. La partie distale des côtes porte de petits tubercules. La morphologie se rapproche de celle des Cardium. Cette dernière est une espèce marine.

Les niveaux BREU 1 et BREU remblai ont fourni une riche faune de gastéropodes. Cette faune ne semble être représentée que par une seule espèce: avec un angle très faible au niveau de l'apex, l'enroulement est rapide ( 5 spires pour $2 \mathrm{~mm}$ de longueur environ) et l'ornementation de la coquille consiste en des côtes parallèles aux stries d'accroissement, une côte étant constituée de 4 à 5 petits tubercules successifs. L'ouverture est siphonostome. Cette espèce ressemble aux Cerithium actuels, genre saumâtre.

Les 3 niveaux échantillonnés renferment une riche faune d'ostracodes. Le niveau BREU 1 contient les espèces Hammatocythere cf. trituberculata, Loxoconcha favata, Hemicyprideis helvetica et Cytheridea pernota. Le niveau BREU 2 contient les espèces Hammatocythere cf. trituberculata, Cytheridea per- nota et Pseudocandona sp.1. L'échantillon prélevé dans le remblais (BREU remblai) renferme les espèces Hammatocythere cf. trituberculata, Eocytheropteron plicatoreticulatum, Cytherella gracilis, Ghardaglaiia cf. pectina, Pterygocythereis ceratoptera, Loxoconcha favata, Hemicyprideis helvetica et Cytheridea pernota.

Les ostracodes récoltés sont majoritairement marins, mais Hemicyprideis helvetica peut vivre en conditions saumâtres. Pseudocandona sp.1 récoltée dans le niveau BREU 2 est le seul véritable ostracode continental, mais qui peut supporter momentanément des variations de salinité.

Les niveaux BREU 1 et BREU remblai ont fourni une faune de foraminifères typiques du Septarienton des synclinaux du Jura. Le niveau BREU 1 a fourni les espèces Quinqueloculina sp.1, Lenticulina sp.2, Lagena sp. et Bolivina beyrichi. L'échantillon BREU remblai a fourni les espèces Quinqueloculina sp.1, Quinqueloculina sp.2, Dentalina sp., Pyrulina lanceolata, Rosalina sp., Melonis affinis et Astrononion sp.

Les niveaux BREU 1 et BREU remblais ont fourni des radioles d'oursins.

De petites dents de poissons téléostéens ont été récoltées dans le niveau BREU remblais.

Les nannofossiles du niveau BREU 1 ont été étudiés par E. De Kaenel. Les fossiles présents sont Chiasmolithus titus, Reticulofenestra dictyoda, ? Rhabdosphaera sp. En plus des formes autochtones (espèces connues de l'Eocène moyen, NP 15, à l'Oligocène inférieur, NP 21), ce niveau contient également des espèces remaniées de l'Eocène et du Crétacé. La datation de ces niveaux par nannofossiles est problématique: en effet, les différentes zones nannoplanctoniques de cette période sont basées sur des disparitions (Hardenbold et al. 1998), ce qui rend les datations difficiles dans les niveaux à forts remaniements comme c'est le cas ici.

\subsection{Le forage de l'Etang (ETA), (coord. 571.474 / 251.036)}

Dans le cadre d'un mandat pour la construction de la route nationale A16, le bureau MFR a été amené à faire un sondage au lieu-dit de l'Etang. Ce forage de $15 \mathrm{~m}$ montre les Gompholithes \& Conglomérats surmontés par le Septarienton (UMM rhénane) (Fig.3).

La base du forage est constituée de marnes rouges très tendres, contenant un banc conglomératique à éléments calcaires arrondis. Ces marnes sont surmontées par un conglomérat à matrice silteuse et à éléments graveleux calcaires arrondis. Dans la base de ce dernier niveau, la matrice est légèrement cimentée. Cette partie inférieure du forage appartient clairement au faciès des Conglomérats de Porrentruy.

$\mathrm{Au}$ dessus vient un petit banc de $70 \mathrm{~cm}$ de silt légèrement sableux, riche en débris coquilliers et en matière organique. Ce banc est considéré comme zone de transition entre les Conglomérats de Porrentruy et le Septarienton.

Finalement le top du forage est constitué par un grand banc, stratifié horizontalement, d'environ $6 \mathrm{~m}$ de silts argileux contenant des niveaux finement sableux et des niveaux riches 


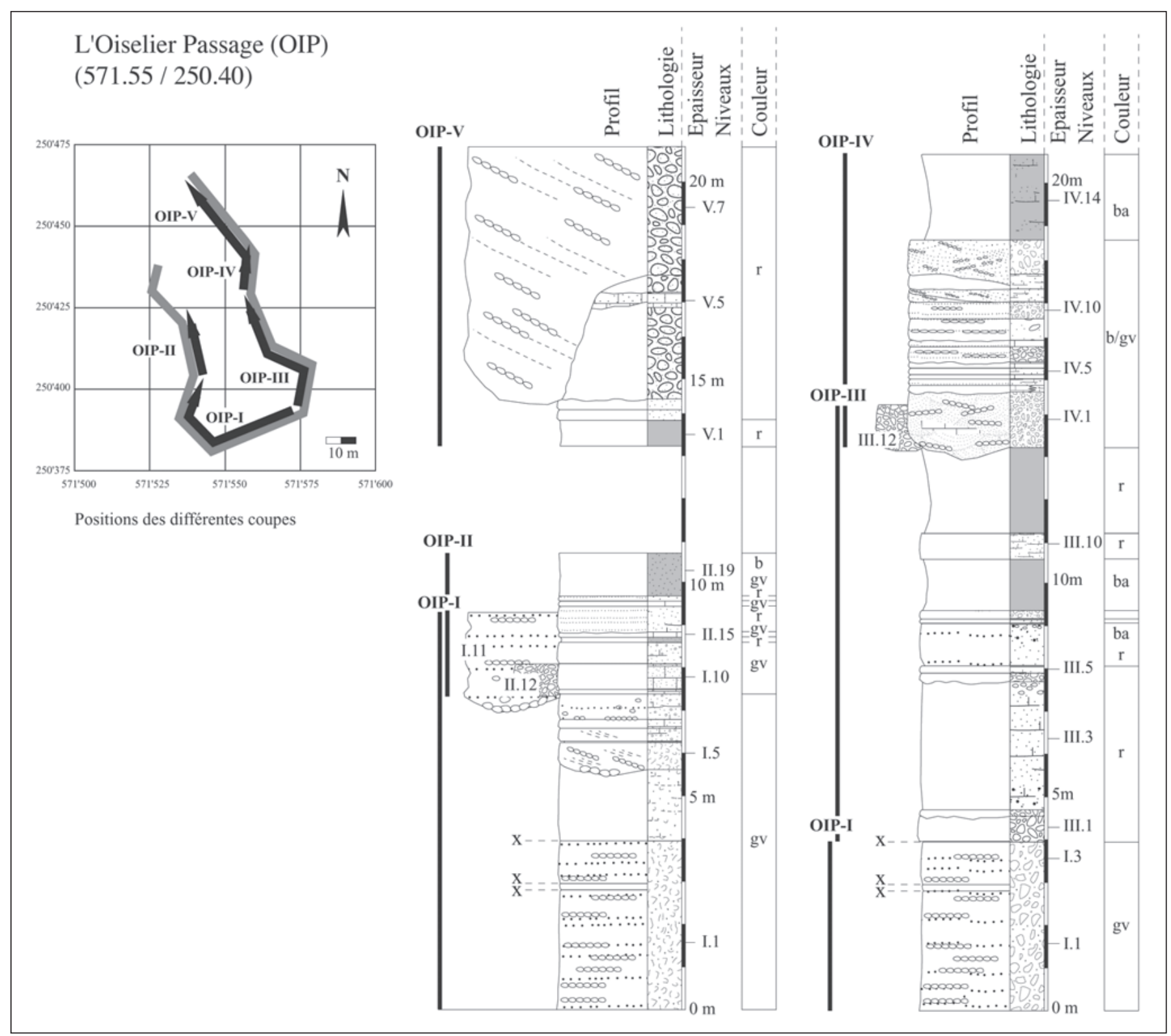

Fig. 4. Logs lithostratigraphiques des coupes de l'Oiselier (Passage à grenouilles-OIP); pour la légende voir Fig. 2.

en matière organique, appartenant au Septarienton. Nous n'avons malheureusement pas eu accès aux résidus de forages (pas d'échantillonnage).

\subsection{La coupe de l'Oiselier (passage à grenouilles) (OIP), (coord. 571.550/250.4)}

Cette coupe se trouve sur le tracé de la future autoroute transjurane A16, découverte à la faveur de la construction d'un passage à grenouilles sous la future autoroute.

Lors de l'excavation, 5 coupes ont été levées. La répartition de ces coupes dans l'excavation d'environ $10 \mathrm{~m}$ sur $10 \mathrm{~m}$ ainsi que les logs lithostratigraphiques sont donnés par la Fig. 4.
La base de la série commence par la section OIP-I, où le pendage général est de $15^{\circ}-20^{\circ}$ vers le NNW. D’une puissance d'une dizaine de mètres, la base de la coupe OIP-I se caractérise par un conglomérat dont les éléments de calcaire sont jointifs dans une matrice gréseuse. La stratification des galets est horizontale. Au-dessus se développe un grès légèrement calcaire où les galets de calcaires mésozoïques ne sont pas jointifs sur une épaisseur de $2 \mathrm{~m}$. Ces dépôts sont interrompus par un petit chenal progradant avec des galets de plus grande taille au contact inférieur. Ce petit chenal contient des éléments de la taille de graviers. Le sommet de la coupe OIP-I correspond à un chenal de plus de $3 \mathrm{~m}$ de puissance à galets calcaires jointifs, contenant lui-même de petits chenaux de graviers d'une épais- 
seur d'environ $1 \mathrm{~m}$. Ces chenaux érodent une série de près de $4 \mathrm{~m}$ de dépôts constitués de calcarénites, grès calcaires, certains niveaux passant à de véritables calcaires. Certains de ces niveaux calcaro-gréseux peuvent être de couleur rouge.

La coupe OIP-II (Fig. 4) se caractérise par des dépôts de calcarénites de $4 \mathrm{~m}$ de puissance. Certains niveaux deviennent légèrement argileux. La couleur de ces bancs horizontaux est majoritairement gris-vert, mais certains sont beiges, d'autres rouges.

La coupe OIP-III (Fig. 4), d'une dizaine de mètres de puissance, se caractérise à la base par un banc d'1 m de conglomérats à galets jointifs sans stratification évidente. Au-dessus se développent $4 \mathrm{~m}$ de calcarénite de couleur rouge, dont la base est un véritable sable. Ces dépôts sont interrompus par un petit chenal relativement plat de $20 \mathrm{~cm}$ d'épaisseur contenant des graviers jointifs. Au-dessus, un banc d'1 m d'épaisseur montre un faciès sableux de couleur rouge et le sommet de ce banc devient bigarré. Ensuite, les dépôts sont représentés par environ $4 \mathrm{~m}$ de marnes rouges ou bigarrés interrompues par un banc interstratifié de calcarénite de $50 \mathrm{~cm}$ de puissance. Le sommet de la coupe OIP-III est constitué de petits chenaux en auge, à stratification entrecroisée, à base ravinante, caractéristiques de rivières en tresse. Ces chenaux contiennent quelques lentilles de calcaire. Ces petits chenaux en auge passent latéralement à des dépôts composés de graviers non jointifs.

La coupe OIP-IV (Fig. 4) montre au-dessus du chenal caractéristique de rivières en tresse, une succession sur $3 \mathrm{~m}$ de calcaires légèrement gréseux interrompus par des petits chenaux plats à stratification horizontale, à base érosive, composés de galets jointifs ou non selon les cas. Ces dépôts calcaires, de couleur beige, très poreux, ont toutes les caractéristiques d'un tuf ou d'un travertin. Ils sont interrompus par un chenal de grès à stratification oblique, puis d'un conglomérat à galets jointifs. Le sommet de la coupe présente $2.50 \mathrm{~m}$ de marnes bariolées.

La coupe OIP-V (Fig. 4) montre, à sa base, $50 \mathrm{~cm} \mathrm{de}$ marnes rouges, surmontées de petits bancs de sable. Avec une base ravinante, se met alors en place un conglomérat à galets jointifs de $2.50 \mathrm{~m}$ d'épaisseur. On peut observer au-dessus un banc calcaire de $20 \mathrm{~cm}$ d'épaisseur, poreux, correspondant à un dépôt travertineux. Ensuite, érodant les dépôts sous-jacents, un conglomérat de plus de $4 \mathrm{~m}$ d'épaisseur montre une stratification oblique d'une trentaine de degrés et prograde vers le nord.

De nombreux galets ont été collectés dans les conglomérats. Ils sont représentés essentiellement par des calcaires du Mésozoïque. Cependant, un niveau conglomératique de la coupe OIP-I (couche OIP-I.11; Fig. 4) a montré près de $5 \%$ de galets de quartzites, de calcaires de couleur rouge, de roches sombres et d'autres roches présentant un aspect granitique. Ce type de galets n'avait jamais été observé jusqu'ici: Duringer (1988) ne signale en effet que des galets de Malm et de Dogger. Différentes lames minces ont été effectuées et révèlent la présence d'amphibolites, de vulcanites, de granitoïdes, de métaclastites, de quartzite et de calcaires à radiolaires (Picot
2002). Les amphibolites présentent, en lame mince, près de $80 \%$ d'amphiboles (actinolite à ferro-actinolite) sur un fond de feldspath représenté par l'albite. Les vulcanites contiennent des plagioclases, des minéraux ferro-magnésiens et un peu de quartz. Cette roche a subi de nombreuses altérations (métamorphisme et hydrothermalisme) car de nombreux minéraux sont rayonnés. Il s'agit d'un porphyre plutôt acide. D'autres types de galets sont des granitoïdes, contenant des plagioclases, des quartz et quelques minéraux ferro-magnésiens. Ces granitoïdes sont légèrement métamorphisés. D'autres galets sont des métaclastiques, à savoir une roche sédimentaire contenant des minéraux provenant d'une roche endogène comme un granite. Les lames minces montrent des plagioclases, des orthoclases pertitiques et du quartz. Cette roche est très altérée. On rencontre également des galets de quartzite. Ces galets blancs ne contiennent que des quartz, provenant d'une pegmatite, d'un filon ou une veine dans une roche endogène. Le dernier type de galet est de couleur rouge et est constitué de calcaires contenant un nombre impressionnant de radiolaires qui ont été calcitisées.

\subsection{La coupe de l'Oiselier (sortie du tunnel) (OIS), (coord. 571.85 / 250.5)}

Sur le tracé de l'autoroute de la Transjurane, au portail ouest du tunnel du Banné, les affleurements montrent le contact entre le Kimméridgien et les Gompholithes et Conglomérats.

La base de l'affleurement est formée par des calcaires du Kimméridgien. Ces calcaires sont traversés par une sorte de conduit contenant des huîtres et des gastéropodes, probablement d'âge tertiaire (Picot 2002). Cette structure, ainsi que les structures similaires observées sur le terrain durant la prospection à OIS et dans le bloc prélevé OIS-I, montrent des structures de «blow holes» (cf. chap. 3 ).

Les calcaires du Kimméridgien sont surmontés d'environ 3 à $4 \mathrm{~m}$ de Gompholithes et Conglomérats.

A la base, on observe un conglomérat à matrice hétérogène calcaire, voire parfois gréseuse, contenant des galets subarrondis à arrondis mal triés (diamètre de 1 à $10 \mathrm{~cm}$ ). Ces galets sont des mudstones contenant peu de fossiles et probablement remaniés du Kimméridgien.

$\mathrm{Au}$ dessus on observe des marnes indurées rouges à concrétions, puis un grès moyen à ciment calcaire.

\subsection{Le forage de la Vauche (RAV), (coord. 572.832 / 251.53)}

Un forage effectué dans la région de la Vauche a été réalisé par Mr Grobet du bureau MFR de Delémont. Il permet d'observer le contact entre les calcaires marneux du Membre du Banné (Kimméridgien moyen) et les Gompholithes \& Conglomérats (Fig.3).

Le contact entre la Molasse et les calcaires du Kimméridgien (Marnes du Banné) se fait de manière nette. La série molassique mesure environ $6 \mathrm{~m}$ et se subdivise en trois parties de 2 m d'épaisseur chacune. 
La première partie se compose d'un niveau relativement homogène de marnes silteuses contenant quelques débris arrondis de calcaire.

Au-dessus, on observe 2 bancs conglomératiques. Le premier montre des graviers calcaires dispersés dans une matrice argileuse. Les graviers sont des mudstones, arrondis à sub-arrondis, dont l'origine est toujours supposée locale. Cependant quelques grains de quartz pourraient avoir une origine différente. Le second banc montre des graviers à cailloux dans une matrice silteuse relativement faible, surmontée d'un petit banc d'argiles silteuses beiges à éléments sub-arrondis.

Ensuite, dans la dernière partie, viennent des argiles grises qui sont bariolées vers le sommet du banc et qui contiennent de nombreux galets de petite taille. Au-dessus de ces argiles grises on peut observer un banc d'argiles rouges bariolées légèrement silteuses. Le banc suivant est formé de calcarénites contenant des éléments calcaires resédimentés dans une matrice silteuse. Le dernier banc avant les dépôts quaternaires est composé d'argiles rouges bariolées légèrement silteuses.

\section{Reconstruction des paléoenvironnements}

Les différentes coupes et forages étudiés permettent une bonne compréhension des paléoenvironnements des dépôts tertiaires de la région de Porrentruy.

La coupe de Bressaucourt Bois Carré (BRE) contient à la fois des faunes marines (foraminifères) et des faunes d'eaux douces (ostracodes). Aucun niveau de la coupe ne contient à la fois les deux types de faunes tertiaires. On est donc en présence d'un milieu côtier situé à la limite du domaine continental et du domaine marin. En plus de l'alternance de faunes marines et continentales, l'existence d'un milieu côtier peut être attesté par la présence de «blow holes» dans la coupe de l'Oiselier (sortie du tunnel, OIS). Les «blow holes» sont des structures perforant des massifs rocheux de type calcaires ou volcaniques, formées par le battement des vagues et des marées sur des barres rocheuses côtières, ou à l'intérieur de karsts côtiers préexistants. Ce type de structures permet donc de tracer la ligne côtière au moment de leur formation. Les «blow holes» de l'Oiselier OIS sont certainement synchrones aux dépôts conglomératiques et ils s'insèrent dans les falaises calcaires du Kimméridgien. Des structures identiques peuvent être observées actuellement, notamment dans les îles calcaires karstifiées du sud-est de la Thaïlande. Dans des calcaires aisément corrodables, la forme des «blow holes» est généralement cylindrique, orientée verticalement ou horizontalement. Leur formation se fait dans un milieu de haute énergie où des gouttelettes d'eau sont projetées en aérosol dans l'air chassé par la vague, puis suivies par la masse d'eau. L'air humide puis l'eau s'engouffrent latéralement ou par le bas et érodent le calcaire en structures fortement déchiquetées. Sur les échantillons observés (OIS), on peut noter la présence de bivalves encore indéterminés accrochés au calcaire, profitants probablement de l'apport de nutriments amenés par l'eau de façon intermittente.
La présence de grands blocs (plus d'1 $\mathrm{m}$ de diamètre) dans les conglomérats montre que l'énergie de ces dépôts est très importante. Seuls des reliefs conséquents peuvent générer ce type d'énergie. On peut expliquer la présence de dépôt de haute énergie avec l'existence à l'époque de falaises d'une dizaine de mètres au moins, entaillées par des canyons où coulent des cours d'eau au régime torrentiel, permettant l'érosion in situ des formations du Mésozoïque sous forme de galets de diamètre important. Le transport est relativement faible mais la création de galets est rapide car les éléments de calcaire roulent les uns sur les autres et s'usent rapidement. Un deuxième fait plaide en faveur de la présence de falaises, c'est l'existence de travertins ou tuf dans les coupes de l'Oiselier passage et la coupe Bressaucourt Bois Carré. Ce type de dépôt calcaire se produit aux émergences de certaines sources, et dans des cours d'eau peu profonds à petites cascades (précipitation des carbonates activée par les turbulences et la perte en $\mathrm{CO}_{2}$ ). Ces cascades seraient dues aux falaises, permettant des dépôts calcaires dans les zones abritées des dépôts conglomératiques de haute énergie des canyons. Ce type de dépôt travertineux est connu actuellement sur les falaises jurassiques de Normandie (France), à Sainte-Marguerite-des Pertes (Calvados) par exemple, où les travertins se développent au niveau des cascades d'eaux douces qui se sont chargées en $\mathrm{CaCO}_{3}$ en traversant les dépôts calcaires jurassiques et qui se jettent sur l'estran.

Les galets des conglomérats issus des canyons se répandent sur l'estran sous la forme d'un delta progradant. Ce caractère progradant est facilement mis en évidence sur la coupe de l'Oiselier-passage (OIP). Le sens de la progradation des conglomérats va du sud vers le nord. Latéralement à ces fan-deltas se trouvent des dépôts gréseux et marneux contenant des foraminifères, caractérisant le domaine marin comme dans la coupe de Bressaucourt Bois Carré (BRE). Le long de la côte et à l'abri des exutoires des canyons, des petits lacs d'eaux douces, alimentés par des cascades, et de faible profondeur, peuvent se développer, permettant l'essor d'ostracodes continentaux. Ces petits lacs se trouvent en haut de plage et se caractérisent par des dépôts marneux. L'alternance de dépôts marins et continentaux montre des fluctuations du niveau marin, dues soit à l'eustatisme, soit à l'accumulation des sédiments, soumettant leur sommet à l'érosion et permettant l'installation de plantes.

Une autre possibilité, non contradictoire, serait la présence de séismes en connexion avec les événements tectoniques. De tels tremblements de Terre sont parfois proposés (par ex. Rothausen \& Sonne 1984) pour expliquer la présence de grands blocs dans les «Untere Meeressande » du Bassin de Mayence.

La présence de galets d'amphibolites, de vulcanites, de granitoïdes, de métaclastites, de quartzites et de calcaires à radiolaires au sommet de la coupe OIP-I montre clairement qu'il existe par intermittence un transport venant du nord, sans doute par la dérive littorale, et dont la source est probablement le massif des Vosges, constitué par des roches endogènes et métamorphiques. La présence d'une dérive littorale implique la création d'un cordon de galets côtier que l'on trouve en haut de plage. 


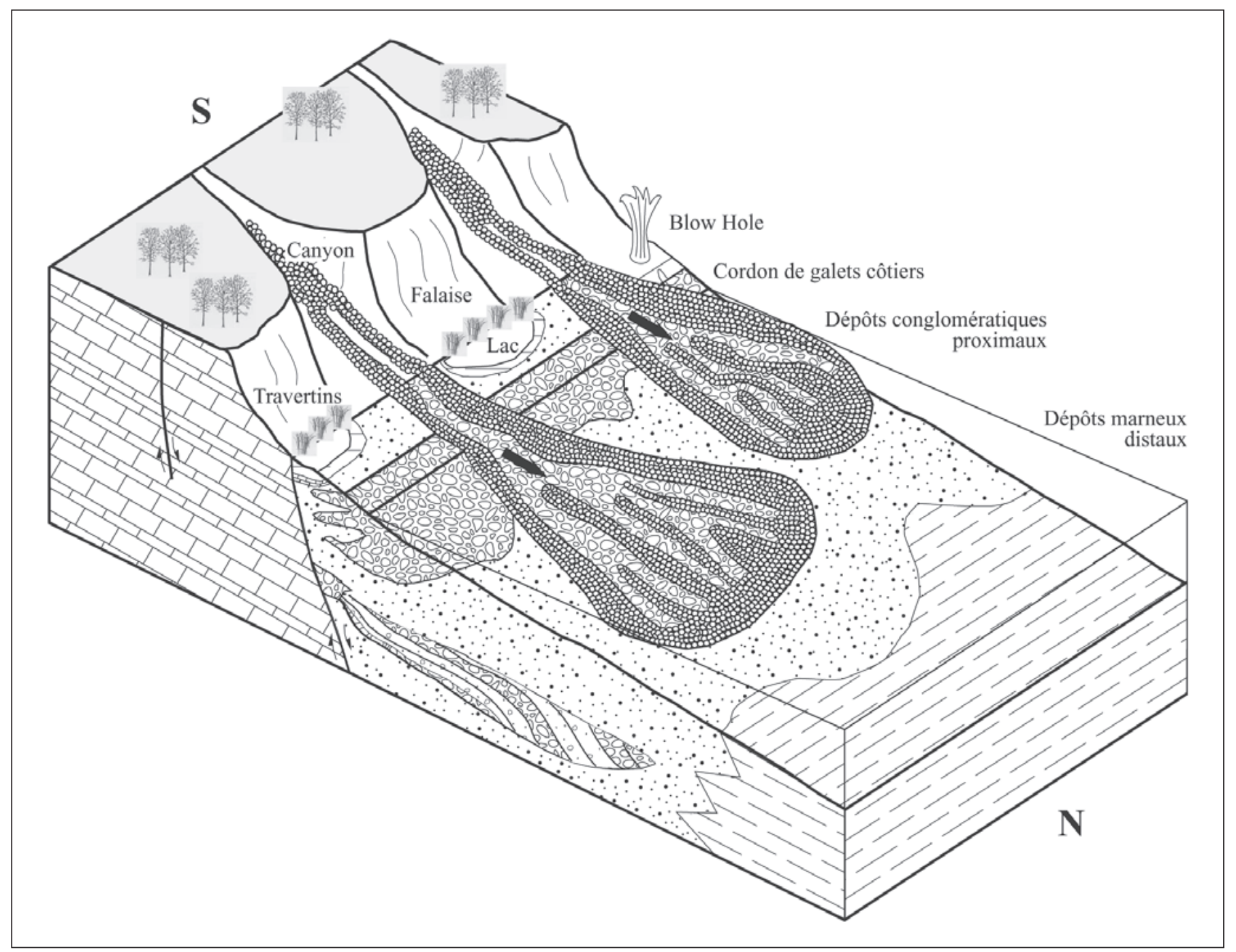

Fig. 5. Reconstitution des différents environnements pour le Groupe des Gompholithes \& Conglomérats de la région de Porrentruy.

Au nord de la coupe Bressaucourt Bois Carré, la coupe Bressaucourt UMM (BREU) montre un faciès marin argileux. En raison de l'absence d'indices stratigraphiques dans les dépôts conglomératiques des coupes de Bressaucourt Bois Carré, de l'Oiselier passage (OIP) et de l'Oiselier (sortie du tunnel) (OIS), on ne peut établir si ces dépôts sont synchrones. Cependant, on peut supposer que ces dépôts argileux marins constituent un faciès plus distal aux dépôts côtiers conglomératiques. Le forage de l'Etang montre que les conglomérats se situent sous les dépôts marins argileux ce qui n'exclue pas des variations latérales de faciès. On peut envisager que la mer rhénane transgressante permet de passer de dépôts côtiers (Groupe des Gompholithes et Conglomérats) à des dépôts marins plus distaux (Septarienton) en passant par des faciès sableux intermédiaires (proches du Meeressand).

La figure synthétique (Fig. 5) montre les différents paléoenvironnements côtiers rencontrés.

\section{Age}

La datation des dépôts conglomératiques de la région de Porrentruy est difficile à établir précisément car les rares faunes recueillies ont une large répartition biostratigraphique (Fig. 6). Cependant, les dépôts similaires de Develier dans le synclinal de Delémont ont pu être datés par la présence de charophytes. Ces charophytes du groupe Harrisichara tuberculata correspondent à un intervalle biostratigraphique situé à cheval sur l'Eocène et l'Oligocène, de la fin du Priabonien jusqu'à la base du Rupélien (Berger 1986 \& 1999, Riveline et al. 1996, Picot 2002). Cependant, il est très difficile d'établir si les dépôts conglomératiques de Develier et de Porrentruy sont synchrones. Les dépôts marins marneux du groupe du Septarienton, situés au dessus des Gompholithes \& Conglomérats comme l'atteste le forage de l'Etang (ETA), ont fait l'objet d'une étude nannoplanctonique sur l'affleurement de Bressau- 


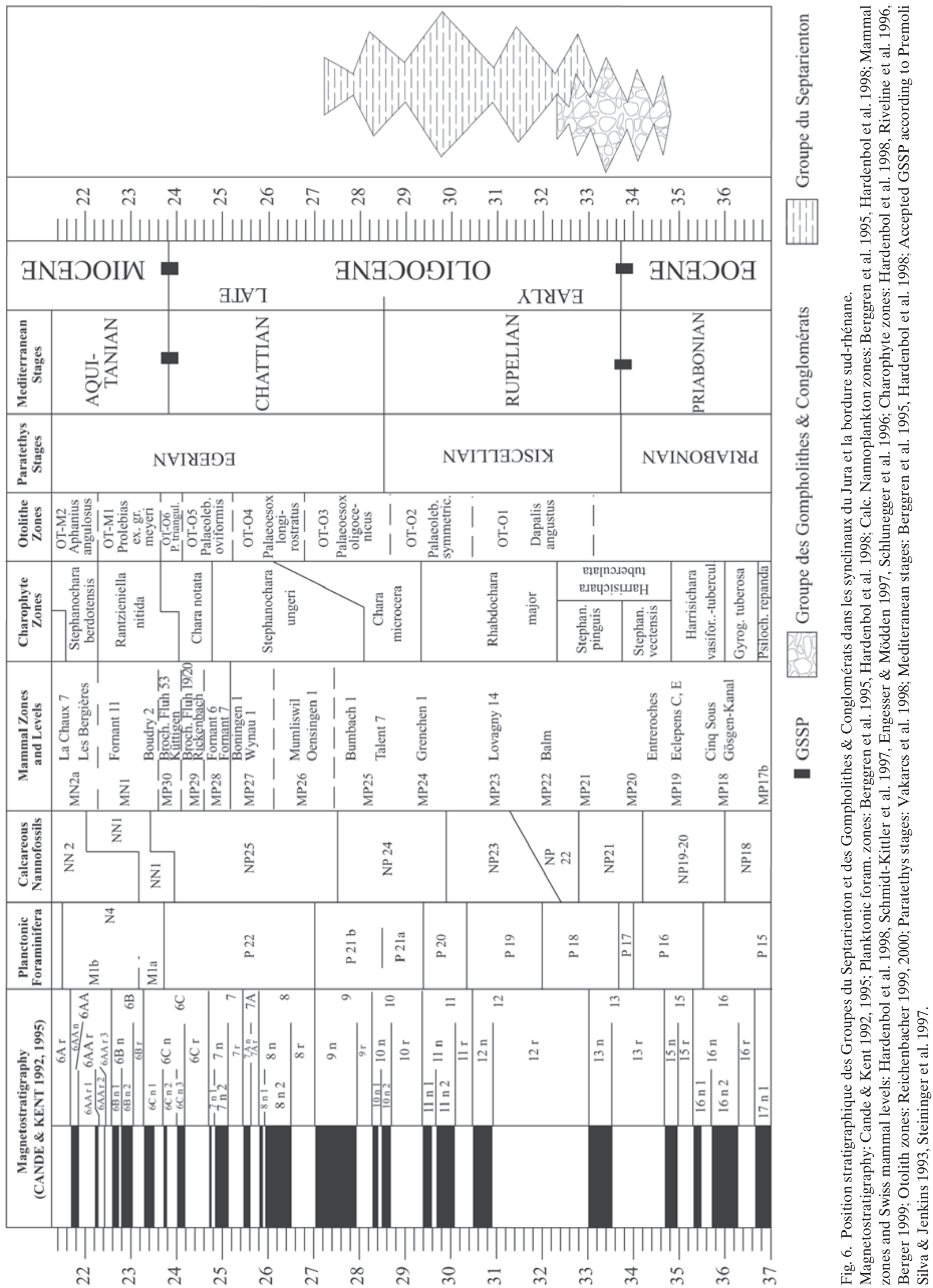




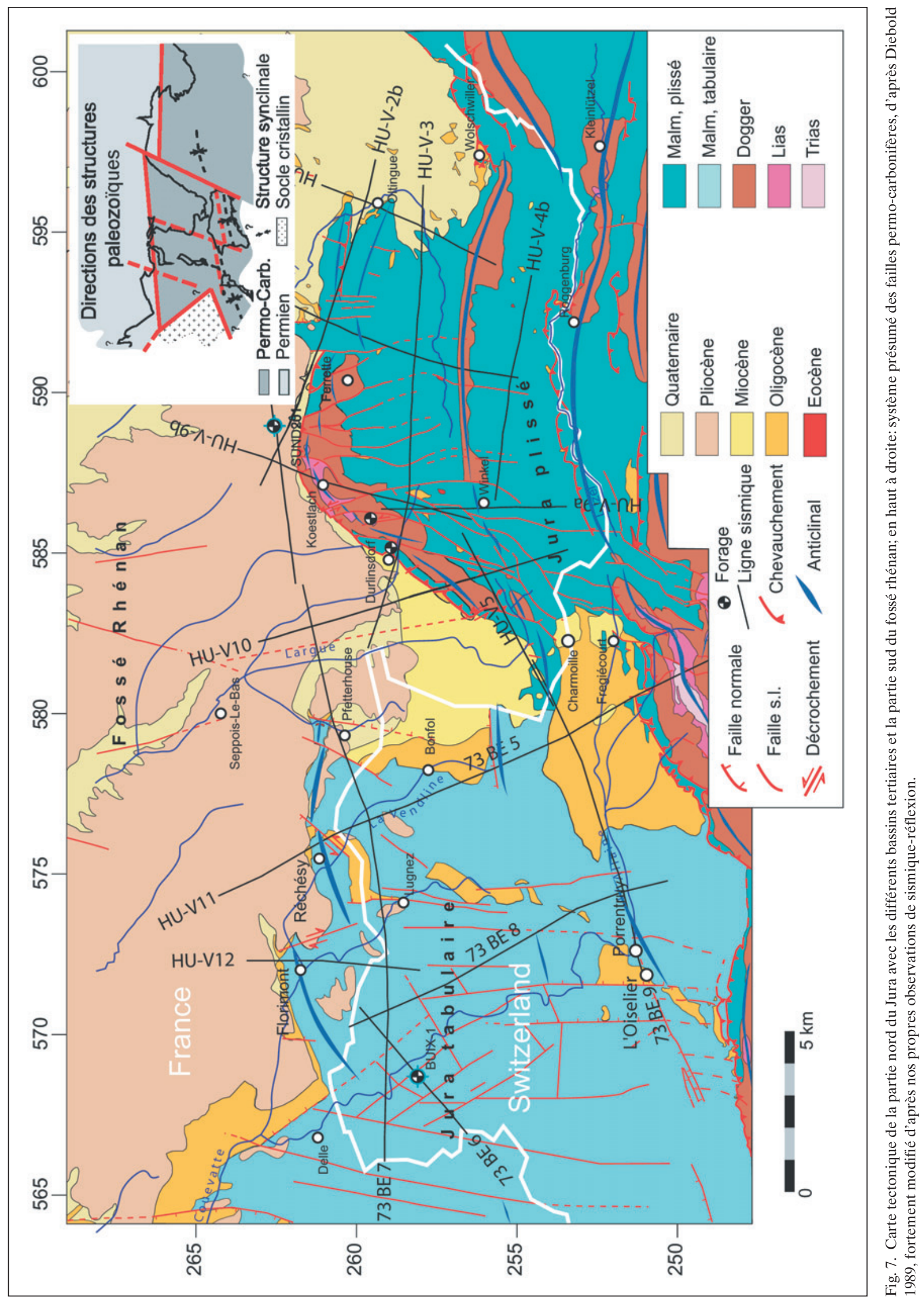


court UMM (Picot 2002). Les espèces récoltés indiquent un intervalle nannoplanctonique allant de l'Eocène moyen (NP 15) à l'Oligocène inférieur (NP 21). Comme le signalait déjà Berger (1992), la datation de ces niveaux par nannofossiles est problématique: en effet, les différentes zones nannoplanctoniques de cette période sont basées sur des disparitions (Hardenbold et al. 1998, Bolli et al. 1985, Berger 1992), ce qui rend les datations difficiles dans les niveaux à forts remaniements comme c'est le cas pour la formation des Septarienton dans le Jura. Dans la plupart des cas, les datations attribuées à NP 21 (Fig. 6) sur la base de la présence de Ericsonia formosa sont contredites par d'autres méthodes de datation biostratigraphique (dinoflagellés) qui donnent des âges plus jeunes basés sur des apparitions d'espèces (Picot 2002).

Le cadastre des gisements de mammifères du Musée de Bâle indique la découverte d'un Aceratherium à l'Oiselier (571.62 / 250.56) et d'un Aceratherium filholi à Bressaucourt. Becker (2003) a révisé ces périssodactyles et rectifie les déterminations en Ronzotherium sp. et Ronzotherium filholi. L'espèce Ronzotherium filholi est caractéristique de l'intervalle mammalien MP 22 - MP 23 (Fig.6), soit la base du Rupélien (Becker 2003). Ainsi, les dépôts conglomératiques de la région se sont mis en place à la base du Rupélien (Oligocène inférieur).

\section{Contexte tectonique de la région de Porrentruy}

La localisation des sédiments paléogènes, ainsi que la forme des bassins tertiaires dans le Jura tabulaire d'Ajoie sont fortement contrôlées par la tectonique liée au rifting du fossé rhénan, affectant toute la succession sédimentaire depuis le socle. Cette région est particulièrement favorable pour étudier les relations entre la tectonique du rift et la sédimentation en raison de l'absence du raccourcissement mio-pliocène lié au plissement du Jura. L'Ajoie se situe à l'extrémité sud du fossé rhénan mais également dans la zone transformante Rhin-Bresse (Rhine-Bresse transform zone RBTZ). Cette zone transformante continentale est liée à l'ouverture du système du fossé rhénan paléogène et du fossé de la Bresse par des mouvements de décrochements sénestres transtensifs dans le socle. Elle s'est formée le long de discontinuités crustales préexistantes héritées de l'orogenèse varisque (Lacombe et al. 1993) et de la transtension post-orogénique qui suivit (de la fin du Carbonifère au Permien).

De nombreux affleurements des Gompholithes \& Conglomérats sont bordés par des failles normales (Diebold et al. 1963, Liniger 1970) ou des flexures, les dernières ayant été trouvées le long de la bordure sud du fossé rhénan (Fischer 1965a, Giamboni et al., soumis). La majorité de ces failles peut être regroupée en deux familles, qui sont particulièrement importantes pour localiser la subsidence paléogène (Fig. 7).

La première famille se rapportant au «Rhénan» selon la littérature (Schumacher 2002) est orientée N jusqu'à (N)NE et est parallèle à la direction du fossé rhénan.

La seconde famille est orientée ENE et a une origine pa- léozoïque terminale. Ces failles sont caractéristiques de l'orientation du système en dépression permo-carbonifère dans les subsurfaces de la partie nord du Jura plissé (Laubscher 1986, Diebold 1989, Diebold \& Naef 1990). Ce système de failles (Fig. 7) est sans doute l'initiateur des mouvements transformants, y compris la zone transformante Rhin-Bresse (RBTZ).

En accord avec le champ de contrainte en vigueur durant l'intervalle principal de rifting du fossé rhénan entre 35 et 28 Ma, les failles «rhénanes» (première famille) sont orientées perpendiculairement à $\sigma 3(\mathrm{E}-\mathrm{W}$ to ESE-WNW; Lacombe et al. 1993) et elles s'accommodent considérablement des rejets verticaux importants. Ce fait devient évident lorsque l'on trace le réflecteur socle-base du Mésozoïque à partir des lignes de sismique-réflexion (Fig. 8). La distension durant l'évolution précoce du rift apparaît le long des failles «rhénanes» (première famille) et de cette façon contrôle l'érosion et le transport des sédiments au moyen de gradins de failles, comme à l'Oiselier (Fig. 7). En combinaison avec le décrochement sénestre lié à la transformante le long des failles orientées ENE, un système de bassins en pull-apart a pu contrôler les dépôts de ces sédiments synrifts précoces (Fig. 8, en haut à droite).

Le contexte tectonique trouve sa réponse sédimentaire par le dépôt de conglomérats proximaux appartenant aux Gompholithes \& Conglomérats et des dépôts plus nordiques de même nature (système de Bourogne et des Conglomérats d'Oltingue), à proximité des failles. Ce genre de scénario peut ainsi expliquer le développement assez rapide de reliefs substantiels permettant la sédimentation de fans alluviaux épais d'une dizaine de mètres.

Ces deux grandes familles de failles décrites sont très importantes pour déterminer la forme des bassins tertiaires dans la région étudiée. Cela est particulièrement bien illustré lorsque l'on s'intéresse à la forme du bassin de Charmoille (Fig. 7). Il est limité à l'Est et à l'Ouest par des failles «rhénanes» présentes en subsurface. Ses limites Nord et Sud sont orientées ENE et sont intimement liées aux segments en dépression permo-carbonifères en subsurface (Fig. 7). Le plissement du Jura au Mio-Pliocène affecte les bordures Est et Sud du bassin: sa bordure Est a été juxtaposée à l'anticlinal de direction NNE et sa bordure Sud a été partiellement chevauchée par des unités jurassiques le long du Mont Terri.

\section{Conclusion}

Les études sédimentologiques et paléontologiques ont permis de reconstituer les différents paléoenvironnements du groupe des Gompholithes et Conglomérats. Ces paléoenvironnements correspondent à des milieux côtiers avec des falaises engendrées par la tectonique distensive rhénane. Ces falaises ont été entaillées par des canyons où se trouvent des rivières au régime torrentiel. Ces rivières qui érodent les dépôts du Mésozoïque créent des galets qui sont déposés sous la forme de deltas marins progradant vers le Nord. A l'abri des exutoires des canyons se développent quelques environnements lacustres et 


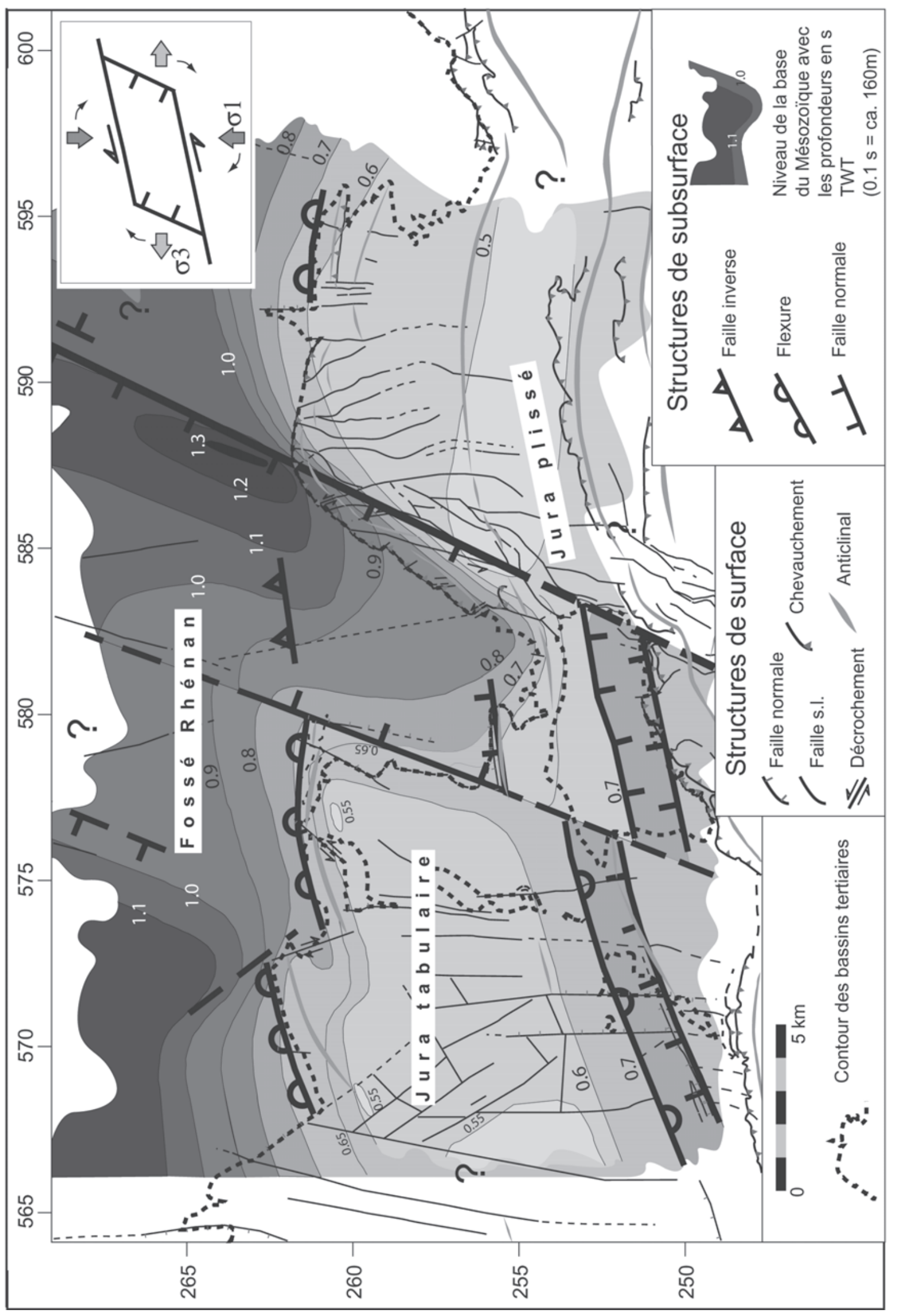

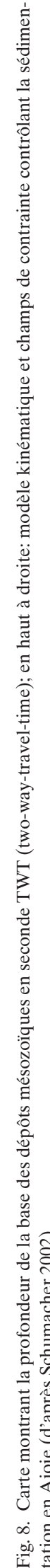


des travertins. Par endroits, la falaise mésozoïque est perforée par des blow-holes.

L'étude tectonique de la région de Porrentruy montre les relations importantes entre les événements tectoniques et les dépôts conglomératiques des Gompholithes et Conglomérats. L'activité distensive du fossé rhénan et l'action de la zone transformante entre le fossé rhénan et celui de la Bresse engendre la création de bassins en pull-apart, et également de gradins de failles, donnant des reliefs substantiels où se développent les Gompholithes et Conglomérats, érodés dans les parties hautes et sédimentés dans les parties basses.

La présence de galets de roches endogènes et effusives dans les Gompholithes \& Conglomérats entraîne une remise en cause de la tectonique sud-rhénane. On considérait généralement que l'érosion du socle des Vosges et de la Forêt-Noire se manifestait dès la limite Oligo-Miocène par le soulèvement sud-rhénan, surtout à l'Aquitanien (Picot et al. 1999, Sissingh 1997 et 1998). Or, les dépôts conglomératiques se sont réalisés à la base du Rupélien. Il faut donc considérer que, déjà au Rupélien, des parties du socle des Vosges subissaient une érosion, sans doute rehaussées sous forme de horsts par de grandes failles, comme le supposait Duringer (1988) pour le versant W du Fossé rhénan.

\section{Remerciements}

Nous tenons à remercier Mr Grobet du bureau MFR de Delémont qui nous a confié les forages de l'Etang (ETA) et de la Vauche (RAV), Mr A. Storni (Fribourg) pour l'échantillonnage des galets, ainsi que Dr. K. Grimm (Mainz) et Dr. E. De Kaenel (Neuchâtel) pour la détermination respectivement des foraminifères et des nannofosssiles. Dr. H. Fischer (Basel) et Prof. B. Reichenbacher (München) nous ont apporté de nombreuses critiques constructives lors de la révision du manuscrit. Cette étude a été réalisée dans le cadre des projets Fonds national FN 2100-052.359.97-1, 20-59220.99 et 81FR-68844 et dans le cadre du projet européen URGENT (EUCOR). K. Ustaszewski a en outre bénéficié du soutien financier du Ministère suisse de l'éducation et de la recherche (BBW), no BBW 99.0567-1.

\section{BIBLIOGRAPHIE}

Apostolescu, V. \& Guernet, C. 1992: Les Ostracodes oligocènes de la région de Forcalquier-Manosque (bassin continental d'Apt, Haute Provence). Rev. Micropaleont. 35/2, 91-115.

Baumberger, E. 1934: Geologischer Führer der Schweiz. Fasc. I. Die Molasse des Schweizerischen Mittellandes und Juragebietes. Schweiz. geol. Ges., Wepf, Basel, 57pp.

BECKER, D. 2003: Evolution paléoécologique et paléoclimatologique de la Molasse du Jura et sud-rhénane: utilisation des Périssodactyles (Mammalia) et des Minéraux argileux. Thèse Univ. Fribourg, Geofocus 9, 327pp.

Berger, J.-P. 1986: Biozonation préliminaire des charophytes oligocènes de Suisse occidentale. Eclogae geol. Helv. 79/3, 897-912.

- 1989: Paleogeographic evolution of the Swiss Molasse basin: a short review. Geologica carpath. 40/1, 9-16.

- 1992: Correlative chart of the European Oligocene and Miocene: application to the Swiss Molasse Basin. Eclogae geol. Helv. 85/3, 573-609.

- 1999: Redefinition of European Oligo-Miocene Charophyte Biozonation. Aust. J. Bot. 47/3, 283-296.

Berggren, W.A., Kent, D.V., Aubry, M.-P., Hardenbol, J. 1995: Geochronology, time scales and global stratigraphic correlation. Soc. econ. Paleont. Mineral. Spec. Publ. 54, 1-386.
BIRKÄUSER, M. 1925: Geologie des Kettenjura der Umgebung von Undervelier (Berner Jura). Verh. natf. Ges. Basel 36, 233-296.

Bolli, H.M., Saunders, J.B. \& Perch-Nielsen, K. (Eds.) 1985: Plankton Stratigraphy. Planktic foraminifera, calcareous nannofossils and calpionellids. Cambridge Earth Science series. Cambridge Univ. Press 1, 599pp.

CAnde, S.C. \& Kent, D.V. 1992: A new geomagnetic polarity time scale for the Late Cretaceous and Cenozoic. J. Geophys. Res. 97, 13917-13951.

CANDE, S.C. \& KENT, D.V. 1995: Revised calibration of the geomagnetic polarity time scale. J. Geophys. Res. 100, 6093-6095.

Diebold, P., 1989: Der Nordschweizer Permokarbon-Trog und die Steinkohlenfrage der Nordschweiz. Vjschr. natf. Ges. Zürich, 133/1, 143-174.

Diebold, P., Laubscher, H.-P., Schneider, A. \& Tschopp, R. 1963: Blatt 1085 Saint-Ursanne. Geol. Atlas Schweiz 1:25’000, Atlasblatt 40, Bundesamt für Wasser und Geologie.

Diebold, P.\& NaEF, H. 1990: Der Nordschweizer Permokarbontrog. Nagra informiert 2, 29-36.

Duringer, P. 1988: Les conglomérats des bordures du Rift cénozoïque rhénan. Dynamique sédimentaire et contrôle climatique. Thèse Univ. Louis Pasteur, Strasbourg, 278 pp.

Duringer, P. \& GALL, J.-C. 1994: Morphologie des constructions microbiennes en contexte de fan-delta oligocène. Exemple du rift rhénan. Palaeogeogr. Palaeoecol. Palaeoclimat. 107, 35-47.

Engesser, B. \& MödDEn, C. 1997: A new version of the biozonation of the Lower Freshwater Molasse (Oligocene and Agenian) of Switzerland and Savoy on the basis of fossil mammals. In: Aguilar, J.P. et al. (Eds.): Actes du Congrès Biochrom'97, Mém. Trav. EPHE, Inst. Montpellier 21, 475-499.

Fischer, H. 1965a: Geologie des Gebietes zwischen Blauen und Pfirter Jura (SW Basel) mit einem mikropaläontologischen und einem paläogeographischen Beitr. geol. Karte Schweiz, N.F. 122, 106 pp.

- 1965b: Blatt 1066 Rodersdorf. Geol. Atlas Schweiz 1:25’000, Erläut. 49, 24 pp.

Freytet, P. \& Plaziat, J.-C. 1982: Continental carbonate sedimentation and pedogenesis - Late Cretaceous and Early Tertiary of southern France. Contrib. Sedimentol. (Füchtbauer, H., Lisitzyn, A. P., Milliman, J. D. \& Seibold, E. Eds.) 12, 213 pp.

Giamboni, M., Ustaszewski, K., Schumacher, M., Schmid, S. \& Wetzel, A. (in press): Plio-Pleistocene Transpressional Reactivation Of Paleozoic And Paleogene Structures. In: The Rhine-Bresse Transform Zone (Northern Switzerland And Eastern France). Int. J. Earth Sci., in press.

Hardenbold, J., Thierry, J., Farley, M.B., Jacquin, T., De Graciansky, P.-C. \& VAIL, P. 1998: Mesozoic and Cenozoic sequence chronostratigraphic framework of European basins. In: DE GRACIANSKY, P.C. et al. (Eds).: Mesozoic and Cenozoic sequence stratigraphy of European basins. Soc. econ. Paleont. Mineral. Spec. Publ. 60, 3-13.

Homewood, P., Allen, P. \& Williams, G.D. 1986: Dynamics of the Molasse Basin of Switzerland. Spec. Publ. int. Assoc. Sedimentol. 8, 199-217.

Lacombe, O., Angelier, J., Byrne, D. \& Dupin, J. 1993: Eocene-Oligocene tectonics and kinematics of the Rhine-Saone continental transform zone (Eastern France). Tectonics 12(4), 874-888.

LAubscher, H. 1986: The eastern Jura: Relations between thin-skinned and basement tectonics, local and regional. Geol. Rdsch. 75(3), 535-553.

LiNiger H. 1925: Geologie des Delsberger Beckens der Umgebung von Movelier. Beitr. geol. Karte Schweiz, N.F. 55, 71 pp.

- 1970: Blatt 1065 Bonfol. Geol. Atlas Schweiz 1:25’000, Karte 55, mit Erläuterungen. Bundesamt für Wasser und Geologie.

PicOT, L. 2002: Le Paléogène des synclinaux du Jura et de la bordure sudrhénane: paléontologie (Ostracodes), paléoécologie, biostratigraphie et paléogéographie. Thèse Univ. Fribourg, Geofocus 5, 240 pp.

Picot, L., Becker, D. \& Berger, J.-P. 1999: Nouvelles données paléoécologiques et biostratigraphiques sur la formation des Calcaires delémontiens («Delsberger Kalke », Oligocène terminal, Jura Suisse). N. Jb. Geol. Paläont. Abh. 214/3, 433-462.

Premoli Silva, I. \& Jenkins, D.G. 1993: Decision on the Eocene-Oligocene boundary stratotype. Episodes 16/3, 379-382. 
ReICHENBACHER, B. 1999: Preliminary otolith-zonation in continental Tertiary deposits of the Paratethys and adjacent areas. N. Jb. Geol Paläont Abh. 214/3, 375-390.

ReICHENBACHER, B. 2000: Das brackish-lakustrine Oligozän und UnterMiozän im Mainzer Becken und Hanauer Becken: Fischfaunen, Paläoökologie, Biostratigraphie, Paläogeographie. Cour. Forschung Senckenberg 222, 1-143.

Riveline, J., Berger, J.P., Feist, M., Martin-Closas, C., Schudack, M. \& SOULIE-Märsche, I. 1996: European Mesozoic-Cenozoic charophyte biozonation. Bull. Soc. géol. France 167/3, 453-468.

RolLier, L. 1893: Etude stratigraphique sur les terrains tertiaires du Jura bernois (partie septentrionale). Eclogae geol. Helv. 4, 1-27.

Rothausen, K. \& SonNe, V. 1984: Mainzer Becken. Sammlung geologischer Führer 79, 203 pp. Berlin, Stuttgart.

Schlunegger, F., Burbank, DW., Matter, A., Engesser, B. \& Mödden, C. 1996: Magnetostratigraphic calibration of the Oligocene to Middle Miocene (30-15 MA) mammal biozones and depositional sequences of the Swiss Molasse basin. Eclogae geol. Helv. 89/2,753-788.

Schmidt-Kittler, N., Vianey-Liaud, M., Mödden, C. \& Comte, B. 1997 New data for the correlation of mammal localitites in the European Oligocene: Biochronological relevance of the Theridomyidae. In Aguilar et al. (Eds.): Actes du Congrès Biochrom'97, Mém. Trav. EPHE, Inst. Montpellier 21, 375-395.

Schneider, A. 1960: Geologie des Gebietes von Siegfriedblatt Porrentry (Berner Jura). Beitr. geol. Karte Schweiz N.F. 109, 72 pp.
Schumacher, M., 2002: Upper Rhine Graben: the Role of Pre-Existing Structures during Rift Evolution. Tectonics 21(1), 1-17.

SissingH, W. 1997: Tectonostratigraphy of the North Alpine Foreland Basin: correlation of Tertiary depositional cycles and orogenic phases. Tectonophysics 282, 223-256.

- 1998: Comparative Tertiary stratigraphy of the Rhine Graben, Bresse Graben and Molasse Basin: correlation of Alpine foreland events. Tectonophysics 300, 249-284.

Steininger, F., Aubry, M.P., Berggren, W.A., Biolzi, M., Borsetti, A.M., Cartlidge, J.E., Catti, F., Corfield, D., Gelati, R., Iaccarino, S., Napoleone, C., Rögl, F., Roetzel, R., Spezzaferi, S., Tateo, F., Villa, G. \& ZeVEnBoOM, D. 1997: The global stratotype section and point (GSSP) for the base of the Neogene. Episode 20/1, 23-28.

Vakarcs, G., Hardenbol, J., Abreu, V.S., Vail, P.R., Varnai, P. \& Tari, G. 1998: Oligocene-Middle Miocene depositional sequences of the central Paratethys and their correlation with regional stages. In: DE GRACIANSKY, P.C. et al. (Eds).: Mesozoic and Cenozoic sequence stratigraphy of European basins. Soc. econ. Paleont. Mineral. Spec. Publ. 60, 209-231.

Manuscrit reçu le 4 février 2003

Révision acceptée le 30 mars 2005

Published Online First November 23, 2005 